PHAGOCYTES, GRANULOCYTES, AND MYELOPOIESIS

\title{
Invasive fungal infection and impaired neutrophil killing in human CARD9 deficiency
}

\author{
Agata Drewniak, ${ }^{1,2}$ Roel P. Gazendam, ${ }^{1}$ Anton T. J. Tool, ${ }^{1}$ Michel van Houdt, ${ }^{1}$ Machiel H. Jansen, ${ }^{2}$ John L. van Hamme, \\ Ester M. M. van Leeuwen, ${ }^{2}$ Dirk Roos, ${ }^{1}$ Emmanuel Scalais, ${ }^{3}$ Carine de Beaufort, ${ }^{4}$ Hans Janssen, ${ }^{5}$ Timo K. van den Berg, ${ }^{1}$ \\ and Taco W. Kuijpers ${ }^{1,6}$ \\ ${ }^{1}$ Sanquin Research, and Landsteiner Laboratory, Academic Medical Center, University of Amsterdam, Amsterdam, The Netherlands; ${ }^{2}$ Department of \\ Experimental Immunology, Academic Medical Center, Amsterdam, The Netherlands; ${ }^{3}$ Division of Pediatric Neurology, Centre Hospitalier de Luxembourg, \\ Luxembourg; ${ }^{4}$ Division of Pediatric Endocrinology, Centre Hospitalier de Luxembourg, Luxembourg; ${ }^{5}$ Division of Cell Biology, Dutch Cancer Institute, \\ Amsterdam, The Netherlands; and ${ }^{6} \mathrm{Emma}$ Children's Hospital, Academic Medical Center, University of Amsterdam, Amsterdam, The Netherlands
}

\section{Key Points}

- Human CARD9 deficiency is characterized by a selective neutrophil killing defect, resulting in invasive candidiasis.

Caspase recruitment domain-containing protein 9 (CARD9) is an adaptor molecule in the cytosol of myeloid cells, required for induction of T-helper cells producing interleukin-17 (Th17 cells) and important in antifungal immunity. In a patient suffering from Candida dubliniensis meningoencephalitis, mutations in the CARD9 gene were found to result in the loss of protein expression. Apart from the reduced numbers of CD4 $^{+}$Th17 lymphocytes, we identified a lack of monocyte-derived cytokines in response to Candida strains. Importantly, CARD9-deficient neutrophils showed a selective Candida albicans killing defect with abnormal ultrastructural phagolysosomes and outgrowth of hyphae. The neutrophil killing defect was independent of the generation of reactive oxygen species by the reduced NAD phosphate oxidase system. Taken together, this demonstrates that human CARD9 deficiency results in selective defect in the host defense against invasive fungal infection, caused by an impaired phagocyte killing. (Blood. 2013;121(13):2385-2392)

\section{Introduction}

Opportunistic invasive fungal infections, such as those with Candida species, are an increasing problem in medicine, as a consequence, among other things, of chemotherapy-induced leukopenia. ${ }^{1}$ The host defense against Candida infections in human subjects appears to rely on innate as well as adaptive immune mechanisms.

It has become clear that, in particular, the responses mediated by the interleukin-17 (IL-17)-producing Th17 subset of T lymphocytes play an essential role in the host immunity against Candida infections. ${ }^{2}$ This is illustrated by a variety of genetic defects associated with the development of T-helper cells producing IL17 (Th17 cells) in patients and an increased susceptibility to candidiasis. ${ }^{3,4}$ The role of IL-17 in the defense against persistent Candida infection has been directly implicated by the identification of IL-17R and IL-17F mutations in pedigrees suffering from chronic mucocutaneous candidiasis (CMC). ${ }^{4,5}$

On the other hand, the innate complement and phagocyte pathways of host immunity against Candida spp in humans have been implied in the clearance of these fungal infections. This is illustrated by the prevalence of invasive Candida spp infections in chronic granulomatous disease (CGD), which is caused by defects in the reduced NAD phosphate (NADPH) oxidase enzyme complex needed for microbial killing, ${ }^{6-8}$ and also by the improved survival upon granulocyte transfusion in case of disseminated candidiasis during chemotherapy-related conditions of prolonged neutropenia. $^{9}$

Although opsonins such as complement are involved in the recognition of Candida by phagocytes, there is evidence that the $\mathrm{C}$ type lectins expressed on the plasma membrane of myeloid cells interact with $\beta$-glucan and mannan residues on the surface of Candida, and thus play an essential role in recognition as well. ${ }^{10-13}$

Exactly which relevant effector functions are triggered through recognition of Candida by lectins, including Dectin-1, Dectin-2, and other related receptors such as Mincle, is not known. Experimental evidence from in vitro studies and in vivo studies in mice suggests that Dectin-1 signals via the tyrosine kinase Syk and via a downstream complex of the cytosolic proteins caspase recruitment domain-containing protein 9(CARD9), Bcl-10, and MALT1 to the transcription factor nuclear factor- $\kappa \mathrm{B}$, which acts as a central regulator in the production of inflammatory cytokines. ${ }^{11}$ Of interest, a recent report has described several cases of CARD9 deficiency in a human family with a history of Candida infections. ${ }^{14}$ Notably, there was evidence that some of the patients within this family not only displayed CMC, but also suffered from invasive Candida infection in the brain. The same study also showed defects in the development of Th17 cells in these patients, linking CARD9 to this pathway of adaptive immunity. However, no studies were
Submitted August 16, 2012; accepted December 30, 2012. Prepublished online as Blood First Edition paper, January 18, 2013; DOI 10.1182/blood2012-08-450551.

A.D. and R.P.G. share first authorship.

The online version of this article contains a data supplement.
There is an Inside Blood commentary on this article in this issue.

The publication costs of this article were defrayed in part by page charge payment. Therefore, and solely to indicate this fact, this article is hereby marked "advertisement" in accordance with 18 USC section 1734.

(C) 2013 by The American Society of Hematology 
performed on myeloid cells, which express CARD9 protein most abundantly. ${ }^{15}$

Here, we describe a patient with CARD9 deficiency who is suffering from chronic invasive Candida infection of the brain, in which we show that - apart from reduced Th17 cell activity - the immune system is more broadly affected. This includes innate defects in inflammatory cytokine production and fungal killing by neutrophils.

\section{Materials and methods}

\section{Cell purification}

The granulocytes and erythrocytes were separated from the mononuclear leukocytes and platelets by centrifugation over isotonic Percoll with a specific density of $1.076 \mathrm{~g} / \mathrm{mL}$. Peripheral blood mononuclear cells (PBMCs) were cultured in Iscove modified Dulbecco medium supplemented with a $10 \%$ fetal calf serum, gentamycine, and $\beta$-mercaptoethanol. Erythrocytes in the pellet were lysed. Granulocytes were washed and resuspended in HEPES $(\mathrm{N}-2-$ hydroxyethylpiperazine- $N^{\prime}$-2-ethanesulfonic acid)-buffered saline solution. ${ }^{16-18}$

\section{Immunostaining and FACS analysis}

After erythrocyte lysis, expression of surface-bound receptors on granulocytes was assayed in total leukocyte samples by flow cytometry (fluorescenceactivated cell sorter [FACS]), with commercially available monoclonal antibodies (mAbs), mouse anti-human Dectin-2/CLEC6A (clone 545943), Dectin-1/CLEC7A (clone 259931) (R\&D Systems, Minneapolis, MN), indirectly labeled with Alexa 488-rabbit-anti-mouse-immunnoglobulin (Ig) (Molecular Probes, Eugene, OR). Samples were analyzed on an LSRII flow cytometer equipped with FACSDiva software (BD Biosciences, San Jose, CA. Cells were gated based on their forward and side scatter, and 10000 gated events were collected per sample.

\section{Western blot analysis}

Analysis of protein expression was performed by western blot. ${ }^{17}$ The following antibodies were used for detection: polyclonal rabbit anti-human CARD9 (Protein Tech Group, Chicago, IL), polyclonal mouse anti-human CLEC4E/MINCLE (Abcam, Cambridge, United Kingdom), monoclonal mouse anti-human CLEC6A/Dectin-2, CLEC7A/Dectin-1 (R\&D Systems), and monoclonal mouse anti-human BCL-10 (clone 151; Invitrogen, Carlsbad, CA).

\section{Lymphocyte activation in vitro}

PBMCs were resuspended in phosphate-buffered saline (PBS) at a concentration of $5 \times 10^{6}$ cells $/ \mathrm{mL}$ and labeled with $0.5 \mu \mathrm{M}$ 5,6-carboxyfluorescein diacetate succinimidyl ester (Molecular Probes, Eugene, OR) as described previously. ${ }^{19,20}$ T-cell proliferative capacity was determined by addition of optimal concentrations of mAbs against CD3 (CLB-CD3/4.E; IgE isotype) \pm CD28 (CLB-CD28/15E8; IgG1 isotype), or purified Candida antigen $(25 \mu \mathrm{g} / \mathrm{mL})$.

\section{Cell stimulation and cytokine measurement}

For IL-17 induction, PBMCs were cultured at a concentration of $2.5 \times 10^{6}$ $\mathrm{T}$ cells per $\mathrm{mL}$ and were stimulated for 72 hours with a combination of antiCD3 and anti-CD28 mAbs.

Where indicated, PBMCs were restimulated with phorbol myristate acetate (PMA, $20 \mathrm{ng} / \mathrm{mL}$; Sigma-Aldrich) and ionomycin $(1 \mu \mathrm{g} / \mathrm{mL}$; Sigma-Aldrich) 6 hours before supernatant collection in the absence or presence of Brefeldin A.

Cytokine concentrations of IL-17 in culture supernatant were analyzed with an enzyme-linked immunosorbent assay (ELISA) using anti-human IL-17A capture mAb (eBioscience; clone eBio64CAP17).
For IL- 6 and IL-1 $\beta$, tumor necrosis factor- $\alpha$ and for IL-8 production, PBMCs $\left(0.25 \times 10^{6}\right.$ monocytes per $\left.\mathrm{mL}\right)$, NB4 or polymorphonuclear leukocytes $(\mathrm{PMN})\left(5.0 \times 10^{6}\right.$ cells $\left./ \mathrm{mL}\right)$ were stimulated overnight with the indicated stimuli. Production of IL-6, IL-1 $\beta$, tumor necrosis factor- $\alpha$, and IL-8 was measured in the supernatants with a Pelikine ELISA (Sanquin Reagents, Amsterdam, The Netherlands) according to the manufacturer's protocol.

\section{Killing of microorganisms}

Short-term microbicidal activity of granulocytes was determined as previously described. ${ }^{21}$ In brief, Escherichia coli (strain ML-35), Staphylococcus aureus (strain 502A), and Candida albicans (stably transfected with green fluorescent protein [GFP]; a generous gift of Dr Alexander Johnson, Department of Microbiology and Immunology, University of California at San Francisco, San Francisco, CA) were grown under aerobic conditions at $37^{\circ} \mathrm{C}$ (bacteria) or $30^{\circ} \mathrm{C}$ (yeast) overnight in Lysogeny broth (LB). Bacteria and yeasts were then collected by centrifugation, washed twice in PBS, and resuspended in HEPES medium. After opsonization $\left(10 \%[\mathrm{v} / \mathrm{v}]\right.$ pooled serum, 15 minutes, at $\left.37^{\circ} \mathrm{C}\right)$, bacteria were added at a ratio of 5:1 neutrophils $\left(4 \times 10^{6}\right.$ cells per $\left.\mathrm{mL}\right)$ and Candida at ratio of 4:1. At the desired time points, $50-\mu \mathrm{L}$ samples were diluted in $2.5 \mathrm{~mL}$ of water $/ \mathrm{NaOH}$ at $\mathrm{pH} 11.0$. At the end of the incubation period, the number of viable microorganisms in each sample was determined by the pour-plate method in LB agar. The colony-forming units were counted after an overnight incubation at $37^{\circ} \mathrm{C}$, and the percentage of killing was calculated. ${ }^{21}$

To assess long-term microbicidal activity of granulocytes, PMNs $\left(5.0 \times 10^{6}\right.$ cells per $\mathrm{mL}$ ) were cultured overnight with the $C$ albicans-GFP, at a yeast: neutrophil ratio of 4:1. The appearance of the hyphenated form of $C$ albicans was assessed with a digital fluorescence microscope (Evos, Westburg, Belgium). This was also determined after addition of granulocyte-colony-stimulating factor (G-CSF) (optimal concentration, $20 \mathrm{ng} / \mathrm{mL}$ ), granulocyte macrophage-CSF (optimal concentration, $10 \mathrm{ng} / \mathrm{mL}$ ), and interferon- $\gamma$ (IFN- $\gamma$ ) (optimal concentration, $100 \mathrm{ng} / \mathrm{mL}$ ) to the overnight culture of neutrophils and Candida conidia.

To determine the PMN killing of $C$ albicans hyphae, conidia (OD: 0.2 , $625 \mathrm{~nm}$ ) were cultured in LB for 16 hours at $37^{\circ} \mathrm{C}$ for formation of a hyphae monolayer, 100 - to $120-\mu \mathrm{m}$ long and confluence. Neutrophils were added for 2 hours in different effector:target $(\mathrm{E}: \mathrm{T})$ ratios, lysed in water $(\mathrm{pH}=11)$, washed in PBS, and incubated for 3 hours with yellow tetrazolium salt MTT (3-(4,5-dimethylthiazol-2-yl)- 2,5-diphenyltetrazolium bromide). The hydrogenases of the functional hyphae can cleave MTT to its purple derivative MTT-formazan, which can be dissolved in isopropanol and quantified by spectrophotometry. The percentage of viable $C$ albicans hyphae was then determined.

\section{Electron microscopy analysis of phagolysosomes}

Purified neutrophils were fixed in $2 \%(\mathrm{w} / \mathrm{v})$ paraformaldehyde with $0.2 \%$ $(\mathrm{w} / \mathrm{v})$ glutaraldehyde and then processed for ultrathin cryosectioning. ${ }^{19}$ Cryosections (50-nm-thick) were cut at $-120^{\circ} \mathrm{C}$ with diamond knives (diatome) in a cryo-ultramicrotome (Leica, Vienna, Austria) and transferred onto carbon/formvar-coated copper grids. The grids were placed on $2 \%(\mathrm{w} / \mathrm{v})$ gelatin plates at $37^{\circ} \mathrm{C}$, embedded in methylcellulose with $0.6 \%$ uranyl acetate, and examined with a CM10 electron microscope (Philips, Eindhoven, The Netherlands). ${ }^{22}$

\section{Statistics}

Graphs were drawn and statistical analysis was performed with GraphPad Prism version 5.00 for Windows (GraphPad Software, San Diego, CA). Data were evaluated by paired, 2-tailed Student $t$ test and 2-way ANOVA with post hoc Bonferroni test. The results are presented as the mean \pm SEM, as indicated. Significance is mentioned when $P<.05$.

\section{Study approval}

The study is performed according to Dutch rules and regulations with respect to the use of human materials from healthy, anonymized volunteers with written informed consent. All experiments were approved by the Medical Ethical Committee of Sanquin Research and the Academic Medical Centre in 
A
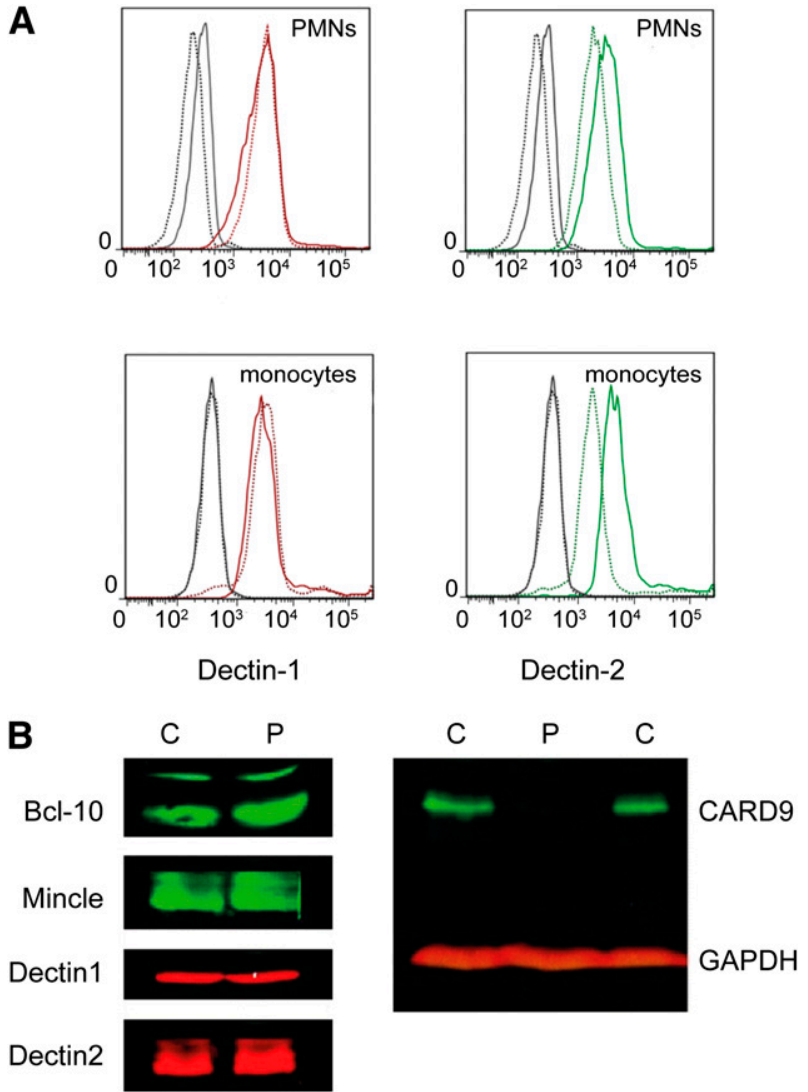

Figure 1. CARD9 deficiency in a patient with systemic candidemia. (A) Surface expression of (left) Dectin-1 and (right) Dectin-2 in control and patient PMNs and monocytes was measured by flow cytometry. Dotted lines represent controls and solid lines patient cells. Isotype controls are shown in gray. Comparable amounts of dectins were detected on the surface of patient and control cells. (B) Western blot detection of C-type lectins (Dectin-1, Dectin-2, and Mincle) as well as Bcl-10 and CARD9 in control and patient neutrophils (indicated as $C$ and $P$, respectively). GAPDH was used as a loading control. Results are representative for 3 independent experiments. GAPDH, glyceraldehyde-3-phosphate dehydrogenase.

Amsterdam which acts according to the Declaration of Helsinki principles (version Seoul 2008).

\section{Supplemental methods}

Supplemental figures and methods are described in the supplemental data available on the Blood website.

\section{Results}

\section{Case report}

We describe an Asian 13-year-old girl who was adopted as an infant. When she first presented at the age of 7 years, she was diagnosed with Candida dubliniensis meningoencephalitis without any obvious underlying risk factors for fungal meningitis. Fever, headaches, behavioral changes, and seizures were the predominant clinical findings. CSF analysis revealed an eosinophilic pleocytosis with increased protein level and decreased CSF glucose. Appropriate antibiotics were initiated adding antimycotics (amphotericine B, followed by fluconazole) which resulted in clinical recovery and normalization of the CSF values. Initial magnetic resonance imaging showed mainly a deep infarction of the left striatum, meningeal enhancement, and mild ventricular dilatation. After 6 months of treatment, the discontinuation resulted in a clinical relapse. Repeated cultures CSF later disclosed $C$ dubliniensis. Clinical signs and CSF pleocytosis resolved within several weeks of restarting combined antifungal therapy (fluconazole, 5-flucytocin). This patient is studied in more detail (Emmanuel Scalais, in preparation).

\section{CARD9 deficiency and mutation analysis}

Surface staining for Dectin-1 and-2 on neutrophils and monocytes, and western blotting for Dectin-1 and -2, Mincle, and Bcl-10 revealed normal levels of these proteins in the lysates of the patient's neutrophils and monocytes (Figure 1; data not shown). However, the CARD9 protein appeared absent (Figure 1B), and this was confirmed with various anti-CARD9 antibodies directed toward different epitopes of the protein. Sequence analysis revealed that the girl was compound heterozygote for 2 previously undescribed mutations c. $214 \mathrm{G}>\mathrm{A}$ and c. $1118 \mathrm{G}>\mathrm{C}$ in $C A R D 9$ (supplemental Figure 1A), resulting in the amino acid substitutions p.Gly72Ser andp.Arg373Pro, which both comprise highly conserved residues in the CARD9 protein in vertebrates (supplemental Figure 1B). These mutations were not found among 100 healthy donors (data not shown). Regarding the medical family history, the adoption agency was able to contact both parents. Although they refused collection of DNA for mutation analysis, clinical data indicated that both parents were over 40 years of age and healthy to date.

\section{T-cell function and Th17 cells}

The presence of AIDS-related invasive Candida infections suggests a role for $\mathrm{CD}^{+}{ }^{+} \mathrm{T}$ cells in such fungal infections. ${ }^{23} \mathrm{We}$ tested T-cell proliferation in response to Candida antigen as well as polyclonal $\mathrm{CD} 3 / \mathrm{CD} 28$ stimulation and detected robust $\mathrm{T}$-cell responses, as was also true for tetanus toxoid, chickenpox, and herpes simplex virus antigen (data not shown).

Of the various $\mathrm{CD}^{+} \mathrm{T}$ lymphocyte subsets, Th17 cells are activated by various interleukins and characteristically release IL-17. This Th17 cell is thought to be a major cell type upon switching from innate to adaptive immunity. ${ }^{11,24}$ In our patient, cytokine profiles from $\mathrm{T}$-cell cultures activated by $\mathrm{CD} 3 / \mathrm{CD} 28$ showed clear induction of various cytokines in the supernatant of these cells (data not shown) but reduced levels of Th17-derived IL-17 (136 \pm 28 and $770 \pm 91$ $\mathrm{pg} / \mathrm{mL}$, patient and controls, respectively; $P<.001$ ), consistent with the data of Glocker et al. ${ }^{14}$

\section{Monocyte and neutrophil function in CARD9 deficiency}

Since CARD9 is primarily expressed in myeloid cells, these cells were explored further. We first investigated the release of inflammatory cytokine release by PBMCs and neutrophils in response to activation by various (un)opsonized pathogens in several independent experiments. This showed the virtual absence in Candidainduced monocytic IL- 6 and IL- $1 \beta$ release in the patient PBMCs, while the response to bacteria (Figure 2A-B) and various Toll-like receptor (TLR) ligands (data not shown) was not or only partly affected. To obtain direct evidence that CARD9 was causative for the defect, we performed lentiviral reconstitution with wild-type CARD9 of hematopoietic stem cells from bone marrow (BM) cultured for 14 days in the presence of macrophage-CSF. The cultured cells were successfully transduced with CARD9 $(80 \%-90 \%$ positive for the reporter nerve growth factor receptor). Partial reconstitution coincided with recovery of BM-derived monocytic cytokines (as 
A

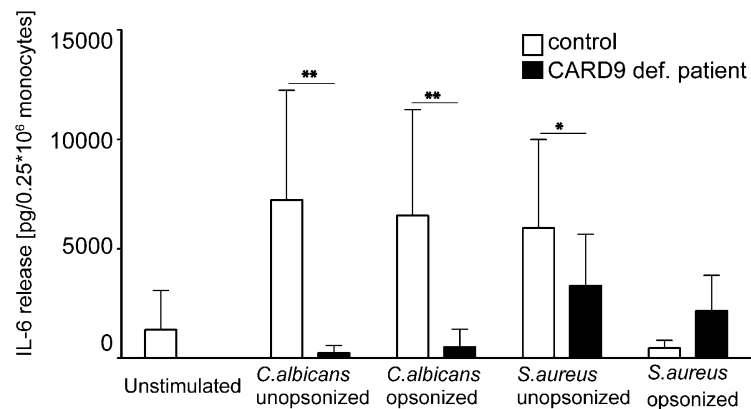

B
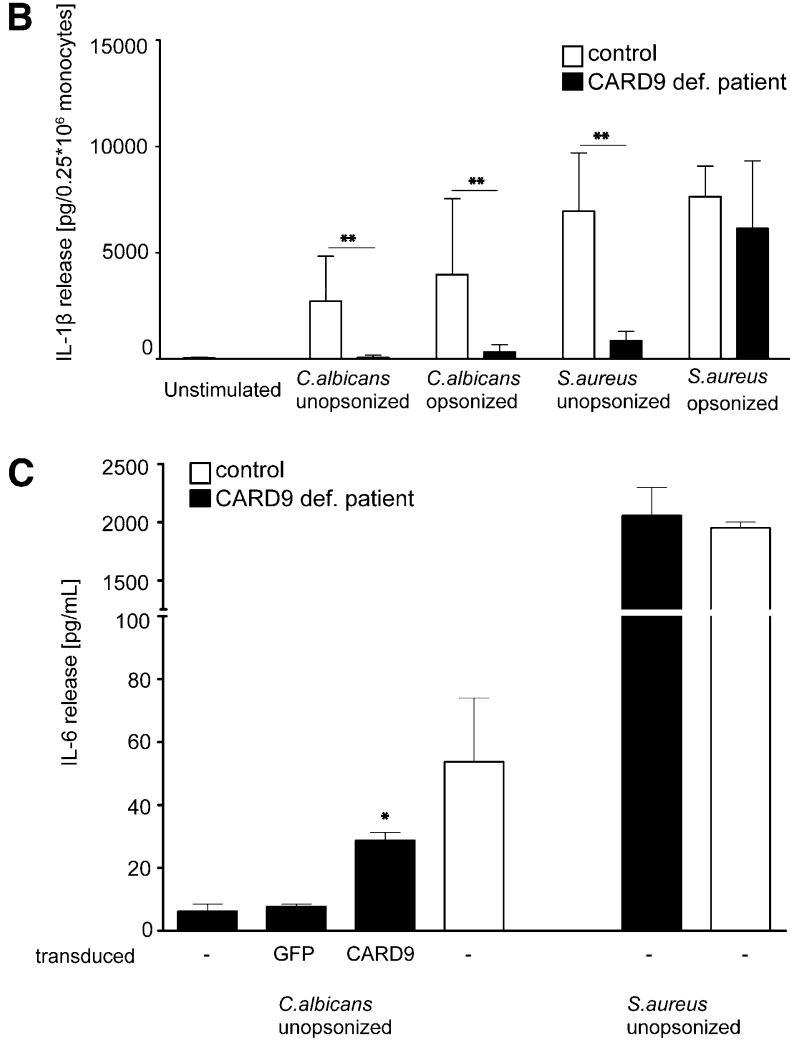

D

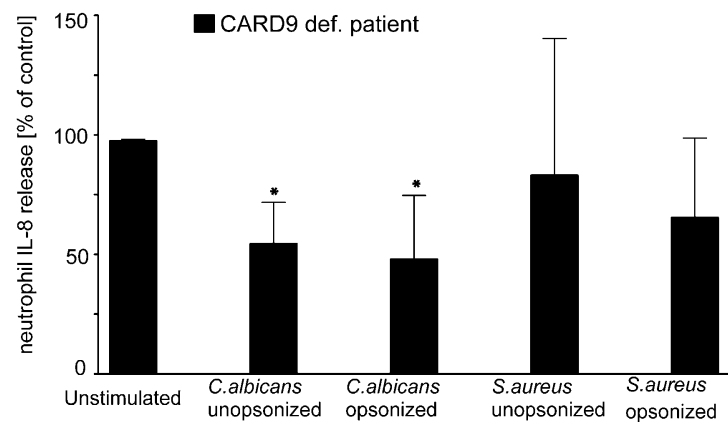

Figure 2. Cytokine production by CARD9-deficient leukocytes and by the patient's $\mathrm{CD}^{+} 4^{+}$hematopoietic stem cells following CARD9 transduction. Leukocytes from the patient and a control subject were incubated overnight with the indicated stimuli. Culture supernatants were collected and the concentrations of IL- 6 and IL- $1 \beta$ by (A-B) PBMCs or (D) IL-8 by neutrophils were assessed by ELISA. To examine the effect of mutated CARD9 on cytokine production, a human wild-type CARD9 complementary DNA (CDNA) was cloned into a lentiviral expression vector. (C) Following transduction, macrophage-CSF-cultured BM-derived monocytic cells of the patient and healthy controls were stimulated with $C$ albicans or $S$ aureus, and the production of IL- 6 was assessed by ELISA. Results represent 2 experiments in triplicate from different BM aspirates of the patient and from healthy controls. Results are means \pm SEM of measurement from 4 to 6 independent experiments; ${ }^{*} P<.01$; ${ }^{* \star} P<.001$.
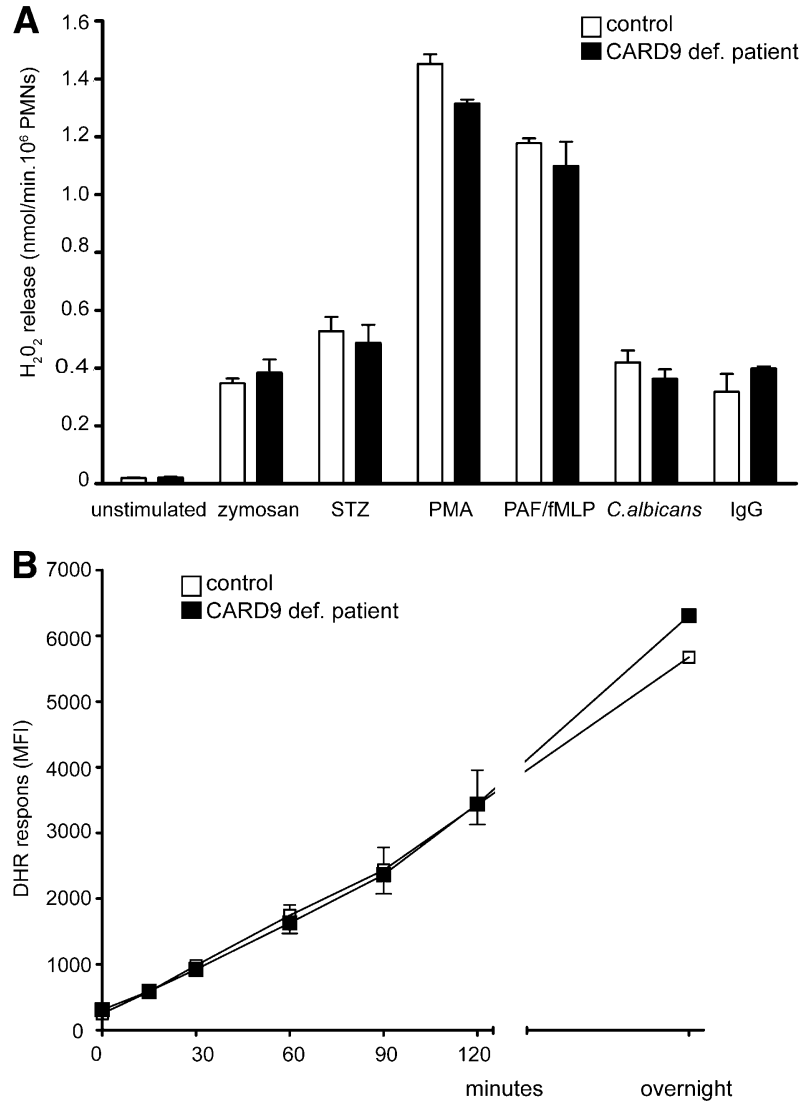

Figure 3. NADPH oxidase activity and phagocytosis of DHR-labeled $C$ albicans in CARD9-deficient neutrophils. (A) To assess the effect of CARD9 deficiency on production of ROS, patient and control neutrophils were stimulated with various stimuli: zymosan, STZ, PMA, PAF followed by fMLP, Candida particles (ratio with neutrophils, 1:4) and heat-aggregated Ig, in the presence of Amplex Red and horseradish peroxidase. Means \pm SEM of at least 3 different experiments. (B) The phagocytosis of DHR-labeled $C$ albicans by the CARD9-deficient and control neutrophils was determined by flow cytometry. Results represent data from 2 different experiments. fMLP, formyl-Met-Leu-Phe; STZ, serum-treated zymosan.

shown for IL-6 upon overnight activation with unopsonized C albicans) (Figure 2C).

We next measured neutrophil-derived IL-8 production in overnight cultures. Although there is considerable interexperimental variation in IL-8 release, ${ }^{16}$ the response of the patient neutrophils to $C$ albicans was consistently decreased (Figure 2D), although not as strongly as the monocyte-derived cytokines. Support for defective cytokine release by CARD9-deficient neutrophils was obtained from experiments with myeloid NB4 cells in which CARD9 was efficiently knocked down (supplemental Figure 3A-B).

In the patient neutrophils, we observed no defects in the immediate induction of NADPH oxidase activity by the various stimuli tested-either primed with platelet-activating factor (PAF) or TLR ligands for subsequent fMLP-induced NADPH oxidase activity, or directly activated by fMLP or the phorbol ester PMA. The same was true for induction of the respiratory burst by the yeast-derived particle zymosan, $C$ albicans particles, or aggregated IgG (Figure 3 ). These findings indicate that the patient cells had a completely normal capacity to generate reactive oxygen species (ROS) and normal recognition and signaling for these immediate responses. ${ }^{17,25}$ Adhesion and chemotaxis upon activation by TLR ligands or chemoattractants such as complement fragment C5a, PAF, or the chemokine IL-8, were all normal (not shown). 
A
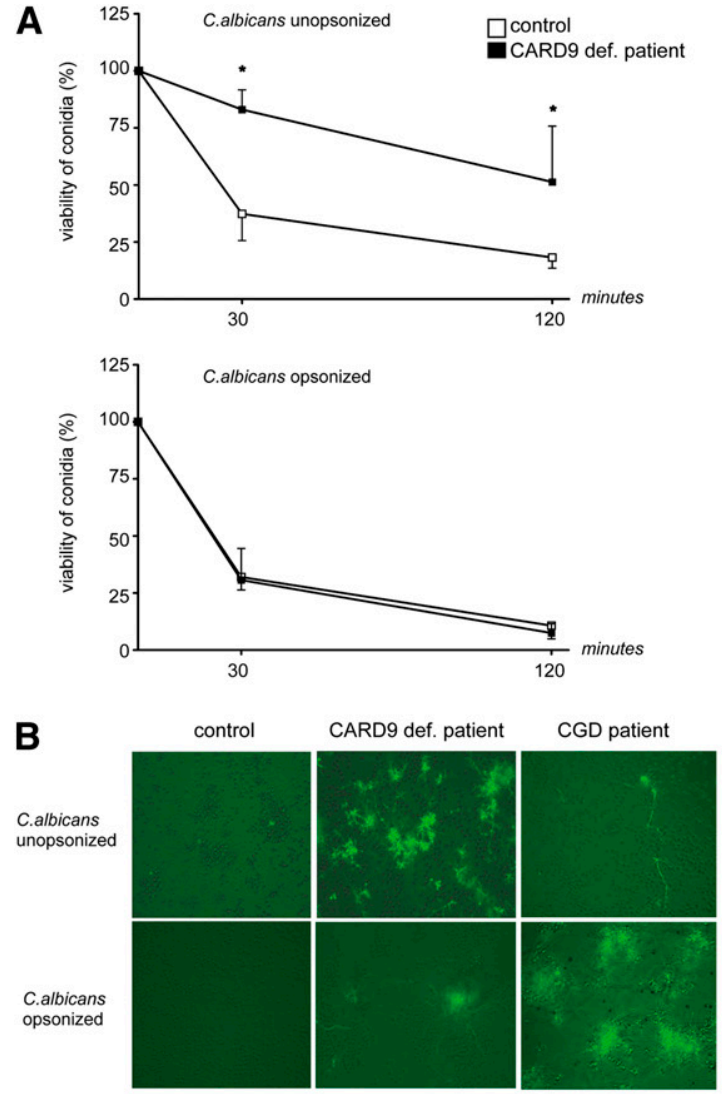

$24 \mathrm{hrs}$
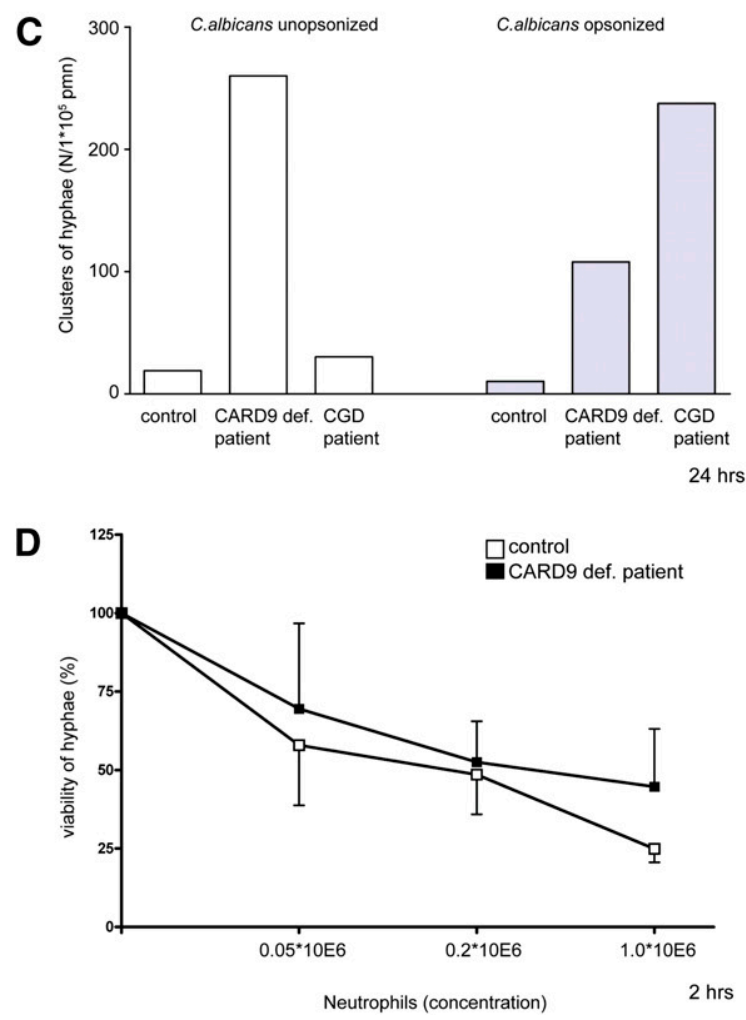

Figure 4. Killing of $\boldsymbol{C}$ albicans by CARD9-deficient neutrophils. (A) Killing efficacy of patient and control neutrophils of unopsonized and opsonized $C$ albicans conidia was assessed by standard colony-forming unit assay. Patient and control neutrophils were incubated with $C$ albicans conidia for 2 hours, and the colonies were counted after overnight incubation of the remaining Candida conidia. Results are
When neutrophil killing capacity was studied, the immediate killing response, within 1-2 hours, by CARD9-deficient cells was significantly impaired with unopsonized $C$ albicans conidia and normal with serum-opsonized conidia (Figure 4A). In contrast, the killing of opsonized gram-positive $S$ aureus or opsonized gramnegative $E$ coli was not different from control neutrophil killing (supplemental Figure 4A). We investigated the cytotoxic response to $C$ albicans. Even though the patient was infected with the philogenetically closely related dubliniensis strain, it has been described that neutrophils have a similar cytotoxic response toward $C$ albicans similar to $C$ dubliniensis in terms of phagocytosis, oxidative burst, and killing. ${ }^{26}$

Importantly, when Candida killing after 18-24 hours incubation was evaluated with CARD9-deficient neutrophils and opsonized $C$ albicans conidia, a clear outgrowth of hyphae was observed which was even more striking with unopsonized $C$ albicans (Figure 4BC, supplemental Figure 5, supplemental Movies 1-4). In contrast to CARD9-dependent killing, such Candida outgrowth was not observed after overnight incubation of neutrophils from CGD patients with unopsonized $C$ albicans (Figure 4B-C). These data demonstrate that a nonoxidative CARD9-dependent and nonredundant mechanism in neutrophils prevents outgrowth of unopsonized Candida. There is a definite role of ROS in Candida killing, but predominantly with the opsonized $C$ albicans conidia. Under these conditions, CGD neutrophils fail to prevent conidia from hyphenation. Of note, CARD9-deficient neutrophils appeared to kill preformed unopsonized $C$ albicans hyphae with similar efficiency as control neutrophils (Figure 4D), indicating that the process of hyphenation itself is insufficiently controlled by CARD9-deficient neutrophils while uptake is intact (supplemental Figure 4B).

Furthermore, measuring the oxidation of dihydrorhodamine (DHR) following the phagocytosis of labeled Candida conidia by neutrophils revealed that patient and control cells showed identical responses (Figure 3B). The normal DHR response means that in the complex process of uptake degranulation and NADPH oxidase activity gets normally activated upon phagolysosome formation. Only after degranulation of the myeloperoxidase from azurophilic granules together with the NAPDH oxidase-derived ROS generation will a DHR signal be generated. Finally, addition of activating factors G-CSF, granulocyte macrophage-CSF, or IFN- $\gamma$, which are known to enhance neutrophil function and survival, ${ }^{27}$ was without any effect on outgrowth of Candida hyphae (not shown).

Because of the normal ROS generation in the patient's neutrophils upon activation by yeast particles, the possibility that the lysosomal killing was dysfunctional was further investigated by ultrastructural analysis. Although neither the release of the content of azurophilic or specific granules nor the NADPH oxidase activity in these

Figure 4 (continued) means \pm SEM of at least 3 different assays; ${ }^{*} P<.05$. (B) Neutrophils from healthy controls, from the CARD9-deficient patient and from CGD patients $(n=9)$, were cocultured overnight with $C$ albicans-GFP and assessed microscopically. Massive outgrowth of the hyphenated form of $C$ albicans-GFP was observed, when cocultured overnight with CARD9-deficient cells. Bright-green fluorescence of hyphae indicates viable Candida. Such outgrowth was not detected when $C$ albicans was cocultured with control neutrophils. (C) Clusters of hyphae were quantified after overnight incubation of $1 \times 10^{5}$ neutrophils with different numbers of Candida conidia. Data are representative for at least 3 experiments. Original magnification, $\times 20$; scale bar, $200 \mu \mathrm{m}$. (D) The capacity of patient and control neutrophils to kill Candida hyphae was determined by MTT assay. Neutrophils in different concentrations were incubated with monolayer of Candida hyphae for 2 hours. The viability of hyphae was assessed by the MTT assay. Results are means \pm SEM of at least 3 different assays. 
A

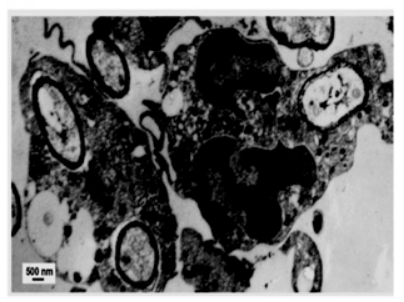

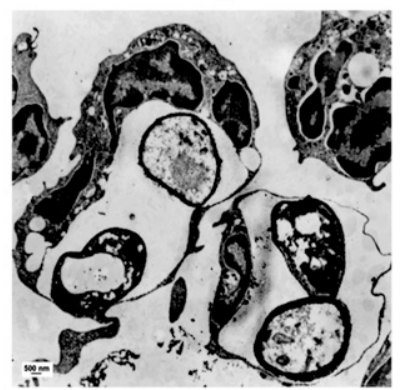

CARD9 def. patient

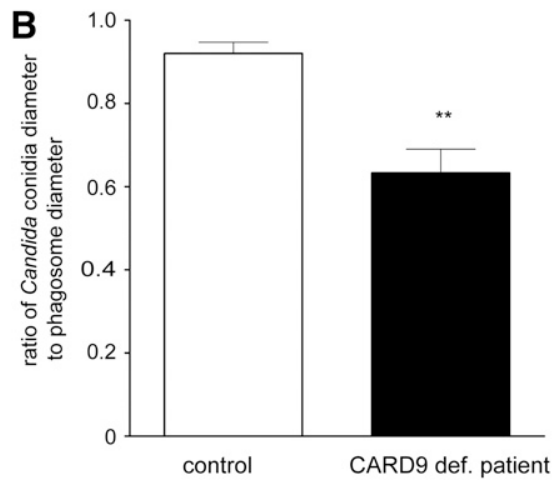

Figure 5. Phagolysosome formation upon uptake of $C$ albicans by human neutrophils. (A) CARD9-deficient and control neutrophils were cocultured overnight with unopsonized $C$ albicans. Ultrastructural analysis was performed by EM. EM pictures showed bulging phagolysosome formation upon uptake of $C$ albicans in the patient neutrophils in contrast to the control neutrophils, obtained in 3 independent experiments. (B) The ratio of Candida conidia diameter to the phagosomal space diameter is significantly reduced in CARD9-deficient neutrophils, taking into consideration that the diameter of the Candida conidia was significantly increased in the case of CARD9 deficiency compared with the control $(1.3 \pm 0.2 \mu \mathrm{m}$ vs $0.8 \pm 0.1 \mu \mathrm{m}$; $P<.05)$. Moreover, the phagosomes of CARD9-deficient neutrophils contained more Candida conidia than the control neutrophils $(1.5 \pm 0.1$ vs $1.1 \pm 0.1$ conidia/ phagosome, $P<.05)$. Taken together, the phagosomes of the CARD9-deficient patient have a strongly enlarged appearance. Results are means \pm SEM of measurement from 3 independent experiments; ${ }^{\star \star} P<.001$.

CARD9-deficient neutrophils was found to be different from control neutrophils (Figure 3A, supplemental Figure 4C), the phagolysosomes in the patient cells following unopsonized Candida albicans, but not $S$ aureus, uptake were abnormal, as clearly demonstrated by electron microscopy (EM) (Figure 5). Taking all these data together, a hitherto unidentified, nonredundant, and relatively selective killing mechanism in neutrophils for fungal organisms is implicated, which depends on the normal function of CARD9.

\section{Discussion}

$S$ aureus and Candida species are the second and third leading causes of bloodstream infections in hospitalized patients. ${ }^{28}$ These organisms jointly cause at least 150000 clinical bloodstream infections resulting in very large health care expenditures and $\sim 40000$ deaths per year in the United States and Europe each. ${ }^{2-31}$ Identification of immune mechanisms of protective adaptive immunity against these organisms is critical to lay the groundwork for development of active preventive and therapeutic strategies against these microorganisms. The induction of cell-mediated immunity to $C$ albicans is one of the main tasks of cells of the innate immune system, and in vitro evidence suggests that integrin $\alpha_{M} \beta_{2}$ (CR3,
Mac-1, CD11b/CD18) is the principal leukocyte receptor involved in recognition of the fungus. ${ }^{18}$

Although $\mathrm{T}$ cells are important in antifungal defense, T-cell reactivity seems to be particularly important for epithelial defense against molds. Among the various subsets of IL-17-producing cells, memory $\mathrm{CD}^{+}$Th17 cells have received most attention regarding their participation in antifungal immune reactivity. ${ }^{2}$ Apart from the release of G-CSF and chemokines to activate neutrophils for microbial clearance, IL-17 also directly induces epithelial cells to produce microbial peptides against, for instance, $S$ aureus and Candida species. ${ }^{32}$ Glocker et al $^{14}$ studied a family with homozygous CARD9 mutation and confirmed the defective Th17 response. In addition, we demonstrate that in human PBMCs the production of IL-6 and IL-1 $\beta$ in response to $C$ albicans is dependent on CARD9. Hereby, we were able to confirm these findings, as described in the card9-knockout mice by LeibundGut-Landmann et al. ${ }^{33}$ The decrease in Staphylococcus-triggered IL-1 $\beta$ in monocytes may suggest a role for CARD9 in this pathway. Indeed, there is evidence that CARD9 signals facilitate the TLR and Nod2 pathways to activate mitogen-activated protein kinase and tyrosine kinase pathways to activate nuclear factor- $\mathrm{\kappa}$ for cytokine production. ${ }^{34}$

We now show that the neutrophils in CARD9 deficiency have a selective defect in Candida killing. Although monocytes and/or dendritic cells may fail to induce Th17 responses in CARD9 deficiency because they do not produce pivotal cytokines such as IL-1 $\beta$ for Th17 differentiation, the killing defect in neutrophils may directly contribute to the invasive nature of the fungal manifestations in CARD9 deficiency and thus contrasts with the Th17associated noninvasive, more localized yeast infections in CMC.

The exact mechanism by which CARD9-defective neutrophils fail to eliminate Candida molds remains to be identified. Some important observations about a potential working mechanism have been obtained. First, the NADPH oxidase activity and, second, the degranulation of azurophilic and specific granules in the patient's neutrophils are normal upon activation (Figure 3, supplemental Figure 4C). This was further supported by the normal oxidative response over time when DHR-labeled Candida conidia were taken up by CARD9deficient neutrophils. In turn, CGD neutrophils which are unable to produce superoxide for oxidative killing were unaffected in their killing activity under conditions where massive Candida conidia outgrowth and hyphenation was observed in the case of CARD9 deficiency. The CARD9-mediated killing mechanism differs from the opsonin-mediated killing pathway, which seems to be completely dependent on fully functional NAPDH oxidase activity as illustrated by the results with neutrophils from CGD patients. These findings are clearly in contrast with a study on BM-derived macrophages from $\operatorname{card9}$-knockout mice, showing less ROS in response to $C$ albicans. ${ }^{35}$

Moreover, the CR3 expression and integrin activity were considered to be normal in all adhesion and chemotaxis assays, as well as in the uptake of zymosan or Candida particles. The ex vivo immediate, early killing response of unopsonized Candida conidia was impaired, but it could be restored upon opsonization with human serum. This indicates that yeast $\beta$-glucan pattern recognition receptors, such as Dectin-1 and/or CR3, depend on CARD9 to activate a selective (nonoxidative) cytotoxic mechanism. This notion is supported by the normal killing of hyphae, which are known to shield their $\beta$-glucans, and are instead recognized by mannan-specific receptors, such as TLR-2, TLR-4, CR3, and Dectin-2. ${ }^{36,37}$

Still, the ultimate Candida evasion from the patient's cells in a prolonged, late killing assay indicates that the rapid and complete elimination of Candida is inadequate and results in escape of 
viable yeast after uptake, resulting in the outgrowth of hyphae. Early after activation of uptake and the intracellular killing reaction, these phagolysosomes tend to swell because the osmotic pressure increases during the breakdown of microbial proteins. ${ }^{37}$ The key machinery required for the export of such osmotically active phagolysosomal breakdown products is unknown, but a role for CARD9 in this process may explain the ultrastructural findings. Alternative explanations may be linked to the dysregulation of autophagy, since CARD9 has been found to associate with Rubicon, a protein known to inhibit autophagosome maturation. ${ }^{38}$ Autophagosomes have been morphologically defined by a quintessential feature: that is, the presence of "double membrane vesicles" as the most critical hallmark of this cellular process. ${ }^{39} \mathrm{We}$ assessed this phenomenon by analyzing EM pictures taken at 2 hours, 4 hours, and after overnight coincubation of neutrophils and Candida strains. In none of the EM pictures, neither in the patient nor in control neutrophils, was the characteristic autophagy signature of double membranes observed (data not shown). In the absence of CARD9, uninhibited Rubicon activity would lead to increased autophagy which seems apparently not to be the case in the patient's neutrophils.

The intact killing response to bacterial strains by CARD9deficient neutrophils indicates that a distinct and nonredundant killing mechanism is induced by the yeast species tested and strictly depends on CARD9 in human neutrophils. In addition, we have studied the inhibition of Aspergillus fumigatus germination. CARD9-deficient neutrophils normally inhibited the germination of A fumigatus (data not shown). This finding suggests that the neutrophil killing defect is specific for Candida species and this is consistent with the susceptibility to Candida infections observed in our patient and those previously reported. ${ }^{14}$

Our findings show that the defect in Candida killing by neutrophils was particularly pronounced in the absence of serum, and this might indeed be relevant in the context of the invasive brain infections observed in our patient and in the patients previously described. ${ }^{14}$ The central nervous system parenchyma is separated from the circulation by the blood-brain-barrier, which limits the access of plasma proteins, including immunoglobulins and complement. ${ }^{40}$ Therefore, in addition to the other potential pathophysiological mechanisms, the central nervous system could be particularly sensitive to Candida infections in the case of CARD9 deficiency. The lack of inflammatory cytokines and neutrophil chemoattractants produced by monocytes and dendritic cells, and possibly also macrophage-like microglia cells, will exaggerate the defect in fungal clearance. The antifungal role of neutrophils is known from clinical experience with intensive cancer treatment. ${ }^{9}$ Our data suggest that hematopoietic stem cell transplantation is the only definite option for curation of CARD9 deficiency.

\section{Acknowledgments}

The authors are most grateful to the parents of the patient for their collaboration, in particular for their agreeing to investigation studies.

A.D., R.P.G., and T.W.K were supported by the Landsteiner Foundation for Blood Transfusion Research (LSBR 1706).

\section{Authorship}

Contribution: A.D., R.P.G., and T.W.K. are the principal investigators and conceived and designed the study; A.D., R.P.G., A.T.J.T., M.v.H., M.H.J., J.L.v.H., E.M.M.v.L., and H.J. performed the experiments; E.S. and C.d.B. assisted in acquisition of the patient samples; D.R. and T.K.v.d.B contributed to the design of the study; A.D. and R.P.G. devised and performed the analyses and wrote the first draft of the manuscript with input from all authors supervised by T.W.K.; and all authors approved the final manuscript revisions.

Conflict-of-interest disclosure: The authors declare no competing financial interests.

Correspondence: Roel P. Gazendam, Sanquin Research, and Landsteiner Laboratory, Plesmanlaan 125, 1066 CX Amsterdam, The Netherlands; e-mail: r.gazendam@sanquin.nl.

\section{References}

1. Richardson MD, Kokki MH. New perspectives in the diagnosis of systemic fungal infections. Ann Med. 1999;31(5):327-335.

2. Cua DJ, Tato CM. Innate IL-17-producing cells: the sentinels of the immune system. Nat Rev Immunol. 2010;10(7):479-489.

3. Heimall J, Freeman A, Holland SM. Pathogenesis of hyper IgE syndrome. Clin Rev Allergy Immunol. 2010;38(1):32-38.

4. Puel A, Cypowyj S, Bustamante J, et al. Chronic mucocutaneous candidiasis in humans with inborn errors of interleukin-17 immunity. Science. 2011;332(6025):65-68.

5. Engelhardt KR, Grimbacher B. Mendelian traits causing susceptibility to mucocutaneous fungal infections in human subjects. J Allergy Clin Immunol. 2012;129(2):294-305, quiz 306-307.

6. van den Berg JM, van Koppen E, Ahlin A, et al Chronic granulomatous disease: the European experience. PLOS ONE. 2009;4(4):e5234.

7. Kuhns DB, Alvord WG, Heller T, et al. Residual NADPH oxidase and survival in chronic granulomatous disease. N Engl J Med. 2010; 363(27):2600-2610.

8. Beauté J, Obenga G, Le Mignot L, et al; French PID Study Group CEREDIH. Epidemiology and outcome of invasive fungal diseases in patients with chronic granulomatous disease: a multicente study in France. Pediatr Infect Dis J. 2011:30(1): 57-62.

9. Safdar A, Hanna HA, Boktour M, et al. Impact of high-dose granulocyte transfusions in patients with cancer with candidemia: retrospective casecontrol analysis of 491 episodes of Candida species bloodstream infections. Cancer. 2004; 101(12):2859-2865.

10. Kerrigan AM, Brown GD. Syk-coupled C-type lectins in immunity. Trends Immunol. 2011;32(4): 151-156.

11. Geijtenbeek TB, Gringhuis SI. Signalling through C-type lectin receptors: shaping immune responses. Nat Rev Immunol. 2009;9(7):465-479.

12. Netea MG, Maródi L. Innate immune mechanisms for recognition and uptake of Candida species. Trends Immunol. 2010;31(9):346-353.

13. Mócsai A, Ruland J, Tybulewicz VL. The SYK tyrosine kinase: a crucial player in diverse biological functions. Nat Rev Immunol. 2010; 10(6):387-402.

14. Glocker EO, Hennigs A, Nabavi M, et al. A homozygous CARD9 mutation in a family with susceptibility to fungal infections. N Engl J Med. 2009;361(18):1727-1735.
15. Bertin J, Guo Y, Wang L, et al. CARD9 is a novel caspase recruitment domain-containing protein that interacts with BCL10/CLAP and activates NF-kappa B. J Biol Chem. 2000;275(52): 41082-41086.

16. Drewniak A, Tool AT, Geissler J, et al. Toll-like receptor-induced reactivity and strongly potentiated IL-8 production in granulocytes mobilized for transfusion purposes. Blood. 2010 115(22):4588-4596.

17. Kuijpers TW, van Bruggen $\mathrm{R}$, Kamerbeek N, et al Natural history and early diagnosis of LAD-1/ variant syndrome. Blood. 2007;109(8):3529-3537.

18. van Bruggen $R$, Drewniak A, Jansen $M$, et al. Complement receptor 3 , not Dectin-1, is the major receptor on human neutrophils for beta-glucanbearing particles. Mol Immunol. 2009;47(2-3): 575-581.

19. Kuijpers TW, Bende RJ, Baars PA, et al. CD20 deficiency in humans results in impaired $T$ cellindependent antibody responses. J Clin Invest. 2010;120(1):214-222

20. Kuijpers TW, ljspeert $H$, van Leeuwen EM, et al. Idiopathic CD4+ T lymphopenia without autoimmunity or granulomatous disease in the slipstream of RAG mutations. Blood. 2011; 117(22):5892-5896. 
21. Drewniak A, Boelens JJ, Vrielink H, et al. Granulocyte concentrates: prolonged functional capacity during storage in the presence of phenotypic changes. Haematologica. 2008;93(7): 1058-1067.

22. Calafat J, Janssen $\mathrm{H}, \mathrm{Knol} E F$, et al. Ultrastructural localization of Charcot-Leyden crystal protein in human eosinophils and basophils. Eur J Haematol. 1997;58(1):56-66.

23. Milner JD, Sandler NG, Douek DC. Th17 cells, Job's syndrome and HIV: opportunities for bacterial and fungal infections. Curr Opin HIV AIDS. 2010;5(2):179-183.

24. Ghoreschi K, Laurence A, Yang XP, et al. Generation of pathogenic $T(H) 17$ cells in the absence of TGF- $\beta$ signalling. Nature. 2010; 467(7318):967-971.

25. van Bruggen R, Drewniak A, Tool AT, et al. Tolllike receptor responses in IRAK-4-deficient neutrophils. J Innate Immun. 2010;2(3):280-287.

26. Peltroche-Llacsahuanga $H$, Schnitzler $N$, Schmidt $\mathrm{S}$, et al. Phagocytosis, oxidative burst, and killing of Candida dubliniensis and Candida albicans by human neutrophils. FEMS Microbiol Lett. 2000; 191(1):151-155.

27. Gaviria JM, van Burik JA, Dale DC, et al. Comparison of interferon-gamma, granulocyte colony-stimulating factor, and granulocytemacrophage colony-stimulating factor for priming leukocyte-mediated hyphal damage of opportunistic fungal pathogens. J Infect Dis. 1999; 179(4):1038-1041.

28. Wisplinghoff $\mathrm{H}$, Bischoff $\mathrm{T}$, Tallent $\mathrm{SM}$, et al. Nosocomial bloodstream infections in US hospitals: analysis of 24,179 cases from a prospective nationwide surveillance study. Clin Infect Dis. 2004;39(3):309-317.

29. Blyth CC, Chen SC, Slavin MA, et al; Australian Candidemia Study. Not just little adults: candidemia epidemiology, molecular characterization, and antifungal susceptibility in neonatal and pediatric patients. Pediatrics. 2009; 123(5):1360-1368.

30. Gudlaugsson O, Gillespie S, Lee K, et al. Attributable mortality of nosocomial candidemia, revisited. Clin Infect Dis. 2003;37(9):1172-1177.

31. Spellberg BJ, Filler SG, Edwards JE Jr. Current treatment strategies for disseminated candidiasis. Clin Infect Dis. 2006;42(2):244-251.

32. Minegishi $Y$, Saito $M$, Nagasawa $M$, et al. Molecular explanation for the contradiction between systemic Th17 defect and localized bacterial infection in hyper-lgE syndrome. J Exp Med. 2009;206(6):1291-1301.

33. LeibundGut-Landmann S, Gross O, Robinson MJ, et al. Syk- and CARD9-dependent coupling of innate immunity to the induction of $\mathrm{T}$ helper cells that produce interleukin 17. Nat Immunol. 2007; 8(6):630-638.

34. Colonna M. All roads lead to CARD9. Nat Immunol. 2007;8(6):554-555.

35. Wu W, Hsu YM, Bi L, et al. CARD9 facilitates microbe-elicited production of reactive oxygen species by regulating the LyGDI-Rac1 complex. Nat Immunol. 2009;10(11):1208-1214

36. Brown GD. Innate antifungal immunity: the key role of phagocytes. Annu Rev Immunol. 2011;29: $1-21$.

37. Reeves EP, Lu H, Jacobs HL, et al. Killing activity of neutrophils is mediated through activation of proteases by K+ flux. Nature. 2002;416(6878): 291-297.

38. Yang CS, Rodgers M, Min CK, et al. The autophagy regulator Rubicon is a feedback inhibitor of CARD9-mediated host innate immunity. Cell Host Microbe. 2012;11(3):277-289.

39. Huang J, Canadien V, Lam GY, et al. Activation of antibacterial autophagy by NADPH oxidases. Proc Natl Acad Sci U S A. 2009;106(15): 6226-6231.

40. Padilla-Docal B, Dorta-Contreras AJ, Bu-CoifiuFanego R, Rey AR. CSF/serum quotient graphs for the evaluation of intrathecal $\mathrm{C} 4$ synthesis. Cerebrospinal Fluid Res. 2009;6:8. 


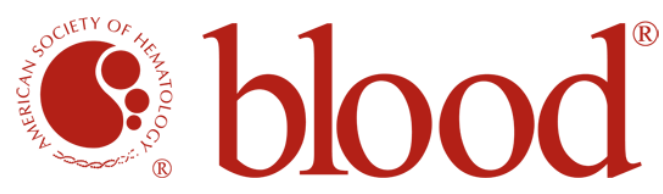

2013 121: 2385-2392

doi:10.1182/blood-2012-08-450551 originally published online January 18, 2013

\section{Invasive fungal infection and impaired neutrophil killing in human CARD9 deficiency}

Agata Drewniak, Roel P. Gazendam, Anton T. J. Tool, Michel van Houdt, Machiel H. Jansen, John L. van Hamme, Ester M. M. van Leeuwen, Dirk Roos, Emmanuel Scalais, Carine de Beaufort, Hans Janssen, Timo K. van den Berg and Taco W. Kuijpers

Updated information and services can be found at:

http://www.bloodjournal.org/content/121/13/2385.full.html

Articles on similar topics can be found in the following Blood collections

Pediatric Hematology (444 articles)

Phagocytes, Granulocytes, and Myelopoiesis (546 articles)

Plenary Papers (479 articles)

Information about reproducing this article in parts or in its entirety may be found online at:

http://www.bloodjournal.org/site/misc/rights.xhtml\#repub_requests

Information about ordering reprints may be found online at:

http://www.bloodjournal.org/site/misc/rights.xhtml\#reprints

Information about subscriptions and ASH membership may be found online at:

http://www.bloodjournal.org/site/subscriptions/index.xhtml

Blood (print ISSN 0006-4971, online ISSN 1528-0020), is published weekly by the American Society of Hematology, 2021 L St, NW, Suite 900, Washington DC 20036.

Copyright 2011 by The American Society of Hematology; all rights reserved. 